\title{
Estudo das propriedades funcionais da hemoglobina de Hoplias malabaricus e Hoplerythrinus unitaeniatus (*)
}

\author{
Austen Riggs ('); Hans Jorgen Fyhn $\left({ }^{2}\right)$; Unni E. H. Fyhn $\left({ }^{2}\right)$; Robert W. Noble $\left({ }^{3}\right)$
}

\begin{abstract}
Resumo
Hemolisados de Hoplias malabaricus e Hoplerythrinus unitaeniatus apresentaram padrões pouco nitidos de hemoglobina com 3 a 4 bandas, res pectivamente, por eletroforesis alcalina em gel de disco. A afinidade da hemoglobina fracionada pelo oxigênio do Hoplerythrinus é cerca de um terço da do Hoplias; os valores do $\mathrm{P}_{50}$ para o Hoplias é cerca de $1,3 \mathrm{~mm}$ de $\mathrm{Hg}$ ( $\mathrm{pH} 6,9$ e $20^{\circ} \mathrm{C}$, . A adição de $1 \mathrm{mM}$ ATP diminui a afinidade de oxigênio de cada hemoglobina 2,6 vezes. Ambas as hemoglobinas apresentam efeito Root e efeito Bohr; $\Delta \log \mathrm{P}_{50} / \Delta$ $\mathrm{pH}=-0,40$ para hemoglobina fracionada no intervalo de $\mathrm{pH} 7$ a 8 . A velocidade de dissociação do oxigênio de cada hemoglobina é semelhante e é cineticamente homogênea com taxas de diminuição constante de 200-250 $\mathrm{sec}^{-1}$ no $\mathrm{pH} 6,2$ para cerca de $25-26$ no pH 7,7 com $1 \mathrm{mM}$ ATP ou sem ele. A reação de combinação do CO para a hemoglobina do Hoplias é cineticamente heterogênea em todos os valores de $\mathrm{pH}$ e para a hemoglobina Hoplerythrinus em pH abaixo 7,5. As fases rápida e lenta explicam cerca da metade da reação observada. A heterogeneidade cinética e máxima em $\mathrm{pH}$ abaixo para ambas as hemoglobinas. A fase rápida para a hemoglobina do Hoplias é cerca de duas vezes mais do que para a hemoglobina do Hoplerythrinus.
\end{abstract}

\section{INTRODUÇÃo}

As duas espécies, Hoplias malabaricus e Hoplerythrinus unitaeniatus, são dois membros relacionados proximamente do primitivo Erythrinidae, já o Hoplerythrinus tem habilidade de respirar ar enquanto o Hoplias não. O classico trabalho de Carter \& Beadle [1931) e Wilimer (1934) mostrou claramente que o Hoplerythrinus respira em águas com baixo teor de oxigênio, confirmando assim o trabalho feito anteriormente por Jobert em 1877 (cf. Carter \& Beadle, 1931). Carter \& Beadle (1931) demonstraram que o oxigênio na bexiga natatória se consumia quando o peixe era privado de ar por duas ou três horas, e Willmer (1934) provou que a respiração aérea se torna obrigatória quando o teor de oxigênio cai abaixo de $1,5 \mathrm{ml}$ por litros aproximadamente, o qual corresponde a um $\mathrm{pO}_{2}$ de cerca de $40 \mathrm{~mm}$ de $\mathrm{Hg}$. Ele também observou que o funcionamento das guelras é sensivelmente dependente do teor de $\mathrm{CO}_{2}$. Num $\mathrm{pCO}_{2}$ acima de $30-35 \mathrm{~mm}$ de $\mathrm{Hg} \mathrm{CO}$, o opérculo está ativamente fechado, e na tensão abaixo de 5-10 $\mathrm{mm}$ de $\mathrm{Hg}$, o teor de $\mathrm{CO}_{2}$ é, na aparência, insuficiente para estimular movimentos operculares. Estas adaptações estão associadas com a habilidade do Hoplerythrinus para sobreviver em lagoas de águas estagnadas durante a época da seca. Embora o Hoplias seja usualmente concentrado em águas correntes, igarapés e rios, também é encontrado em pequenas lagoas estagnadas. Por ser incapaz de respirar ar, tem que utilizar nestas condições a fina camada oxigenada da superfície da água. Willmer (1934) indicou que os Hoplias jovens também sobrevivem em condições de baixo teor de oxigênio com as bocas na superfície e têm uma aparência e hábitos tão diferentes que as pessoas do local chamam-na por um nome diferente. Temos estudado as propriedades funcionais das hemoglobinas do Hoplias e Hoplerythrinus como um primeiro passo para determinar a extensão em que as hemoglobinas destes dois peixes proximamente relacionados podem contribuir para as adaptações.

(*) - Versão original inglesa publicada em Comp. Biochem Physiol. 62 A (1). 1979.

(1) - Department of Zoology, University of Texas at Aus tin, Austin, Texas 78712, U.S.A.

(2) - Institute of Zoophysiology, University of Oslo, Blindern, Oslo 3 NORWAY.

(3) - Department of Medicine and Biochemistry, State University of New York, Buffalo, New York 14215, U.S.A. 


\section{MATERIAL E MÉTODOS}

Espécimes de Hoplias malabaricus e Hoplerythrinus unitaeniatus foram coletados em novembro e dezembro de 1976 durante a expedi. ção na R/V "Alpha Helix" numa área de cerca de $50 \mathrm{~km}$ acima do encontro do rio Amazonas (rio Solimões) com o rio Negro. Espécimes de Hoplias (pesando 390-740 g) foram apanhados à rede perto da saída do lago de Janauacá. Os espécimes de Hoplerythrinus (pesando $72-190 \mathrm{~g}$ ) foram comprados de pescadores. O periodo da coleta foi o fim da estação seca quando as águas estavam em nível mais baixo.

O sangue para a eletroforesis de hemoglobina foi obtido por punção cardíaca, usando-se heparina em $1,7 \%$ de $\mathrm{NaCl}$ como anticoagulante. $\mathrm{O}$ hematócito foi determinado no material fresco, com o sangue misturado em tubos de micro-hematócrito e centrifugado os glóbulos vermelhos foram lavados três vezes em 10 volumes de $1,7 \%$ de $\mathrm{NaCl}$ contudo 1 $\mathrm{mM}$ de Tris, $\mathrm{pH} 8,0$. As células foram lizadas em 3 volumes de $1 \mathrm{mM}$ de Tris $\mathrm{pH} 8,0$ por 1 hora a $\mathrm{O}^{\circ} \mathrm{C}$. $\mathrm{O}$ hemolisado foi levado a $0,1 \mathrm{M}$ em $\mathrm{NaCl}$ e centrifugado a $28.000 \times \mathrm{g}$ por 15 minutos a $4^{\circ} \mathrm{C}$. A hemoglobina para o estudo funcional foi preparada similarmente, exceto que o sangue foi obtido da veia caudal.

A eletroforesis de disco em gel de poliacrilamida alcalina $(\mathrm{pH} 8,9,7,5 \%$ gel foi feita de acordo com Davis (1964) e Ornstein (1964). As amostras de hemoglobinas foram diluídas no tampão da curva superior contendo $0.1 \mathrm{M}$ de $\beta-$ mercaptoetanol e ditionito de sódio sendo borbulhado com o $\mathrm{CO}$ e aplicado no gel. Foi usada como padrão de mobilidade a albumina do soro bovino. Os geis foram corados por 3 horas com $0,25 \%$ "Brilliant blue R" (Sigma) e descorados por difusão. Os geis foram analisados a $560 \mathrm{~nm}$, usando-se um analisador de gel Gilford acoplado a monocromador Beckman DU e acoplado a um registrador Taran ww 600. O peso molecular das cadeias de hemoglobinas desnaturadas foram determinadas por eletroforesis em gel de dodecil sulfato de sódio (SDS) de acordo com Weber e Osborn (1969) exceto, que a incubação da solução foi $6^{*} \mathrm{M}$ em uréia e $0,1 \mathrm{M}$ em $\beta$-mercaptoetanol. Transferina humana, albumina de soro bovino, $\propto$-quimiotripsinogeneo-A e mio- globina na de baleia foram usadas como padrões de peso molecular.

O hemolisado foi separado dos fosfatos orgânicos por cromatografia em uma coluna de Sephadex G-25 equilibrada com $1 \mathrm{mM}$ de Tris, pH 8,0, e também por passagem através de coluna deionizadora descrita por Garlick et al. (1978). Todos os experimentos utilizaram tampões Bis e Tris de força iônica 0,05 . $O$ efeito do ATP foi medido pela adição de 1 mM de ATP no tampão.

O equilíbrio do oxigênio foi medido pelo método de Allen et al., (1950) e Riggs \& Wolbach (1956) e o resultado foi calculado como o descrito por Nagel et al. (1965). A solução foi desoxigenada com água saturada de argônio livre de oxigênio, a qual foi passada através de tonômetros contendo as soluções. Os espectros das soluções foram determinados com espectrofotômetro Beckman modelo DB acoplado ao registrador. Os tonômetros tinham cubetas pyrex de $1 \mathrm{~cm}$ fundidos aos mesmos. Todas as medidas cinéticas foram feitas com um aparelho "stopped-flow" do tipo descrito originalmente por Gibson e Milnes (1964) a $20^{\circ} \mathrm{C}$. As constantes cinética apresentada são aos mínimos quadrados ajustados para as primeiras $65 \%$ das reações observadas. As análises cinéticas das reações bifásicas foram realizadas como descrito por Galdames-Portus et al. (1978).

A cinética da dissociação do oxigênio foi medida por variação de $\mathrm{pH}$, procedimento descrito por Noble et al. (1950). Hemoglobina oxigenada em Tris $1 \mathrm{mM}, \mathrm{pH} 8,0$, foi misturada com uma solução de ditionito de sódio em tampão de força iônica 0,1 no $\mathrm{pH}$ desejado. A concentração final da hemoglobina foi aproximadamente $30 \mu \mathrm{M}$ (heme equivalentes). A reação foi acompanhada a 560 até $540 \mathrm{~nm}$.

A cinética de combinação do monóxido de carbono com a hemoglobina desoxigenada foi medida misturando-se soluções de hemoglobina em tampões de força iônica 0,1 e pH desejado com a solução contendo a concentração conhecida, aproximadamente de $85 \mathrm{uM}$, de monóxido de carbono dissolvido em água. Depois da mistura, a concentração de hemoglobina foi aproximadamente $3 \mu \mathrm{M}$ em heme. A reação foi acompanhada a 420 até $435^{\prime \prime} \mathrm{nm}$. 


\section{RESULTADOS E DISCUSSÃO}

A eletroforesis em gel de disco dos hemolisados de amhns Hoplias a Hoplerythrinus apresentou padrões pouco nítidos de bandas múltiplas com mobilidade eletroforética menor que a $\mathrm{Hb}$. A humana aumentando a concentração da acrilamida no gel para $10 \%$ não melhorou significativamente a resolução. Padrões idênticos de hemoglobina foram encontrados em hemolisados de peixe de diferentes localidades. Hoplias apresentou 3 bandas nos geis com $60 \%$ de concentração de hemoglobina o meio da banda e $20 \%$ em cada uma das outras. Reischl (1976) tinha relatado que dois componentes da hemoglobina do Hoplias formam polímeros dependentes da oxidação. Tais polímeros não poderia ser vistos nos geis descritos aqui por causa da presença do $0,1 \mathrm{M}$ -mercaptoetanol. Hoplerythrinus tem padrão mais complexo com cerca de $80 \%$ da concentração de hemoglobina no meio da banda dupla. $\mathrm{O}$ peso molecular das cadeias de hemoglobina desnaturadas do Hoplias e Hopleythrinus foi estimado ser 14.400 a 14.700 , respectivamente em SDS. Estes valores são semelhantes aos encontrados para cadeias de hemoglobinas humanas desnaturadas, 14.600 . Valores de hematócritos para o Hoplias e Hoplerythrinus foram $31 \pm 6 \%$ e $32 \pm 2 \%$ (média $\pm \mathrm{D}$. P. $\mathrm{N}=5$ ), respectivamente. Os resultados das medidas do equilíbrio do oxigênio, resumidas nas tabelas I e II, mostram que a afinidade pelo oxigênio da hemoglobina do Hoplerythrinus corresponde aproximadamente a um terço da hemoglobina do Hoplias a pH 7-8. A pressão de oxigênio para $50 \%$ de saturação, $\mathrm{P}_{50}$, é cerca de $4,1 \mathrm{~mm}$ de $\mathrm{Hg}$ para a hemoglobina do Hoplias a $20^{\circ} \mathrm{C}$ e pH 6.93. O fator de Bohr médio, $\Delta \log \mathrm{P}_{50} / \Delta \mathrm{pH}$, é aproximadamente $-0,40$ para ambas as hemoglobinas no intervalo de $\mathrm{pH}$ 7-8. Por outro lado, o efeito de $1 \mathrm{mM}$ ATP a pH 6,9 é idêntico para cada hemogglobina diminui cerca de 2,6 vezes. A pH 8 , a afinidade da hemoglobina pelo oxigênio para Hoplias não é atingida pelo ATP enquanto a hemoglobina do Hoplerythrinus decresce cerca de $27 \%$.

O valor do coeficiente de Hill, $n$, e cerca de 1,5-1,6 para ambas as hemoglobinas a $\mathrm{pH}$ $6,9^{\circ}$ e este valor não é mudado pela adição de $1 \mathrm{mM}$ ATP. O valor de $n$ é quase a unidade para a hemoglobina do Hoplias, porém permanece como 1,5 para a hemoglobina do Hoplerythrinus. Entretanto, depois da adição de $1 \mathrm{mM}$ ATP a $\mathrm{pH} 8$, o valor de $n$ é diminuído substancialmente na hemoglobina do Hoplerythrinus enquanto esta adição não tem efeito no valor de $n$ obtido para a hemoglobina do Hoplias.

TABELA I - Sumário do equilíbrio de oxigênio da hemoglobina do Hoplerythrinus unitaeniatus a

\begin{tabular}{lllll}
\hline pH & ATP & $\log \mathrm{P}_{50}$ & $\mathrm{n}$ & $\frac{\log \mathrm{P}_{90}}{\mathrm{pH}}$ \\
& & & & \\
$5.9 \mathrm{~b}$ & - & 1.8 & - & \\
6.9 & - & 0.61 & 1.5 & \\
8.0 & - & 0.17 & 1.5 & \\
6.9 & + & 1.02 & 1.6 & \\
8.0 & + & 0.28 & 1.9 & -0.68 \\
\hline
\end{tabular}

(a) Condiçōes destos experiências:

$200 \mathrm{C}$, tampāo, 0.05 forşa iônica Bis-tris pH abaixo de 7 , 0.05 força iônica Tris pH acima de 8.0. Concentração de ATP $1 \mathrm{mM}$ concentraçăo de hemoglobina $\simeq 40 \mu \mathrm{M}$ (heme).

(b) Estes experimentos foram feitos como se segue:

A hemoglobina foi desoxigenada em $1 \mathrm{~nm}$ Tris, $\mathrm{pH} 8,75$, depois foi suficientemente desoxigenada em $0.2 \mathrm{M}$ Bis-tris de $\mathrm{pH} 5.9$ foi adicionado para baixar $0 \mathrm{pH}$ para 5.9. 0 tampāo final Bis-tris foi de 0,05 de força iônica. Injeção de uma aliquota de ar resultou em único ponto, $Y=c o m \log P=1.73 ;$ e $\log P_{50}$ foi estimado em valores de $n$ compreendidos $1,0<{ }^{n}<1,5$. O espectro da oxiemoglobina foi obtida pela adiçáo de Tris sólido, e equilibrado com ar com alto $\mathrm{pH}$.

TABELA II - Sumário do equilíbrio de oxigênio da hemoglobina do Hoplias malabaricus a

\begin{tabular}{lrrrr}
\hline pH & ATP & $\log \mathrm{P}_{50}$ & n & $\frac{\Delta \log \mathrm{P}_{50}}{\Delta \mathrm{pH}}$ \\
& & & & \\
6,9 & - & 0,01 & 1,6 & -0.40 \\
8,0 & - & $-0,35$ & 1,1 & \\
9,0 & - & $-0,23$ & 1,1 & \\
$6,1 \mathrm{~b}$ & + & 1,14 & 0,7 & \\
6,9 & + & 0,52 & 1,5 & \\
8,0 & + & $-0,35$ & 1,1 & \\
\hline
\end{tabular}

(a) Condiçōes foram descritas na tabela 1

(b) Oxigênio a 1 ATM foi usado para oxigenar a amostra. 


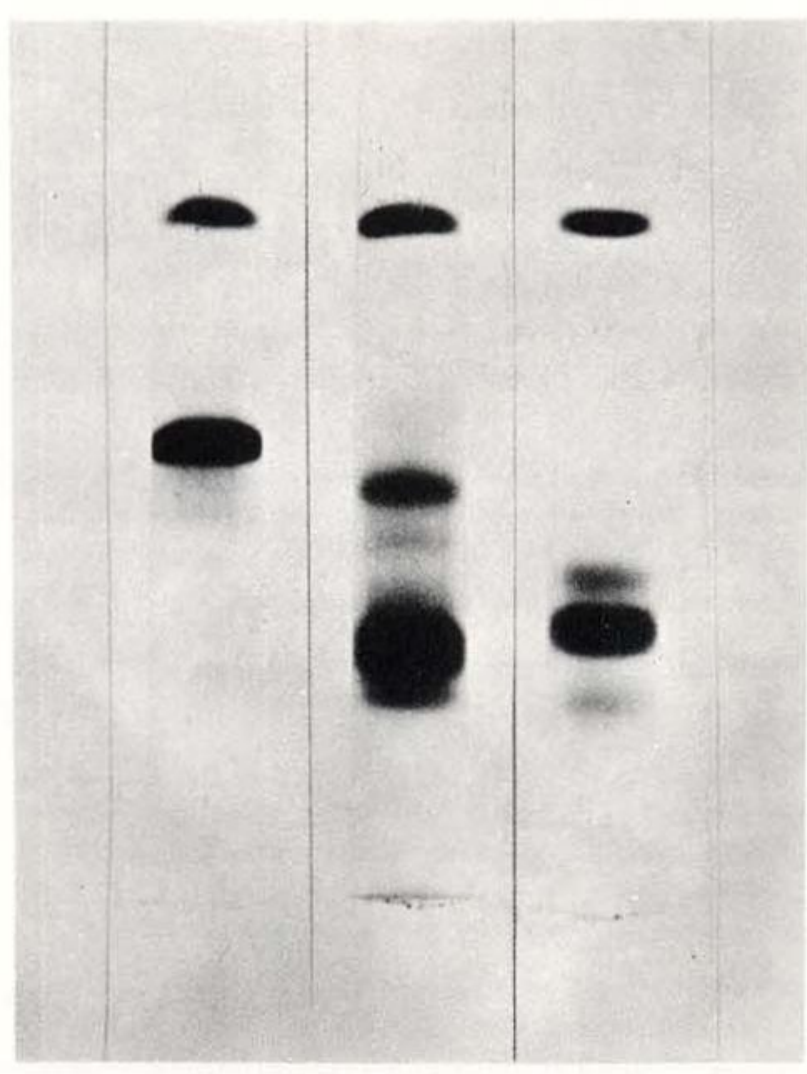

Fig. 1-Poliacrilamida em disco de gel de hemo. lisados de Hoplias malabaricus (esquerda), Hoplerythrinus unitaeniatus (centro), e homem (direita). As bandas restantes em cada gel é de albumina de soro de Boi. O anodo é a base.

A adição de $1 \mathrm{mM}$ ATP diminui o valor aparente do fator de Bohr, $\Delta \log \mathrm{P}_{50} / \Delta \mathrm{pH}$, de $-0,40$ para $-0,68$ (hemoglobina do Hoplerythrinus) e de $-0,40$ para $-0,79$ (hemoglobina de Hoplias) no intervalo de $\mathrm{pH} 7-8$. Estas mudanças não são usuais para a hemoglobina dos teleósteos.

Ambas as hemoglobinas têm efeito Root, porém dados extensivos não foram obtidos a baixo $\mathrm{pH}$ porque cada uma dela não se mostraram sensiveis à oxigenação da meta hemoglobina a baixo $\mathrm{pH}$. $\mathrm{O}$ vaior do $\mathrm{P}_{\mathrm{so}}$ da hemoglobina do Hoplerythrinus foi estimada em cerca de $63 \mathrm{~mm}$ de $\mathrm{Hg}$ a pH 5,9 (veja tabela I). Este experimento, que foi repetido, depende da desoxigenação e alto $\mathrm{pH}$, a adição de tampão desoxigenado para abaixar o $\mathrm{pH}$, seguindo-se de injeção de uma única alíquota de ar. Imediatamente depoîs desta injeção, seguindo o equilíbrio e medição, o tonômetro foi aberto, Tris sólido foi adicionado e o espectro resultante da oxiemoglobina foi medido. O tempo total de exposição da hemoglobina ao $\mathrm{pH}$ baixo foi em torno de 11-12 minutos. Períodos de tempo mais longos (30-40 minutos) conduzem inevitavelmente para a não aceitação de grandes quantidades de metaemoglobina $(50 \%$ ou mais). Se admitirmos que o coeficiente de Hill, $n$, é 1,0 , o grau de saturação a um $\mathrm{pO}_{2}$ de $150 \mathrm{~mm}$ de $\mathrm{Hg}$ (ar) poderá ser cerca de $70 \%$. Do mesmo modo, se o valor de $n$ for alto como 1,5 (valor improvável) o nível de oxigenação poderá ainda ser em tơrno de $75 \%$.

Semelhantemente, na presença de $1 \mathrm{mM}$ ATP, a hemoglobina do Hoplias tem muito mais afinidade pelo oxigênio em $\mathrm{pH}$, próximos de 6 do que a hemoglobina do Hoplerythrinus na ausência de fosfatos orgânicos. A pH 6,1 o valor $\mathrm{P}_{50}$ é apenas $13,8 \mathrm{~mm}$ de $\mathrm{Hg}$, e o valor de $n$ é apenas 0,7 . Estes valores indicam um nível de oxigenação em torno de $83 \%$ a um $\mathrm{pO}_{2}$ de $150 \mathrm{~mm}$ de $\mathrm{Hg}$. Os índices de dissociação do oxigênio para a hemoglobina do Hoplias e Hoplerythrinus foram medidos a três $\mathrm{pH}$ em presença e ausência de $1 \mathrm{mM}$ ATP. Embora estas preparações de hemoglobina contenham múltiplos componentes, as reações parecem ser cineticamente homogêneas e cada uma poderá ser descrita por uma constante de velocidade única. Estas constantes velocidades são ploteadas em função do $\mathrm{pH}$ na fig. 2. As hemoglobinas do Hoplias e Hoplerythrinus apresentam-se muito semelhantes referentes a estas constantes. ATP tem mui pequeno efeito na velocidade de dissociação de oxigênio exceto a pH 6,9 em que na ausência de ATP a constante de velocidade para a hemoglobina do Hoplias é consideravelmente menor desnaturadas do Hoplias e Hoplerythrinus foque as encontradas por outros sistemas. A alta dependência do $\mathrm{pH}$ existem em todos estes sistemas: a constante de velocidade aumenta 10 vezes quando $\mathrm{pH}$ diminui de 7,8 para 6,3.

A reação do monóxido de carbono com a hemoglobina do Hoplias, em contraste com a dissociação de oxigênio é cineticamente heterogênea a todo $\mathrm{pH}$ examinado, com ATP ou sem ATP. Dois processos cinéticos podem facilmente ser discernidos, cada um respondendo aproximadamente pela metade do total da mudança na absorbância. Por suas dependências nos comprimentos de ondas foi claro que 
ambos os processos foram reações de combinação com monóxido de carbono. A constante reação de segunda ordem para as duas fases foram computadas e esboçadas na fig. $3 \mathrm{em}$ função do $\mathrm{pH}$. A fase rápida tem um índice constante cerca de $5 \times 10^{5} \mathrm{M}^{-1} \mathrm{sec}^{-1}$ e é inalterada pelo $\mathrm{pH}$ ou $1 \mathrm{mM}$ ATP. A fase lenta é dependente do $\mathrm{pH}$ na faixa de 6,1 a 7,1 porém permanece inalterado quando o $\mathrm{pH}$ é aumentado além de 8,2. O único efeito significativo do ATP observado é até $\mathrm{pH} 6,1$ na fase lenta o qual é diminuído cada vez mais pela adição de $1 \mathrm{mM}$ de ATP.

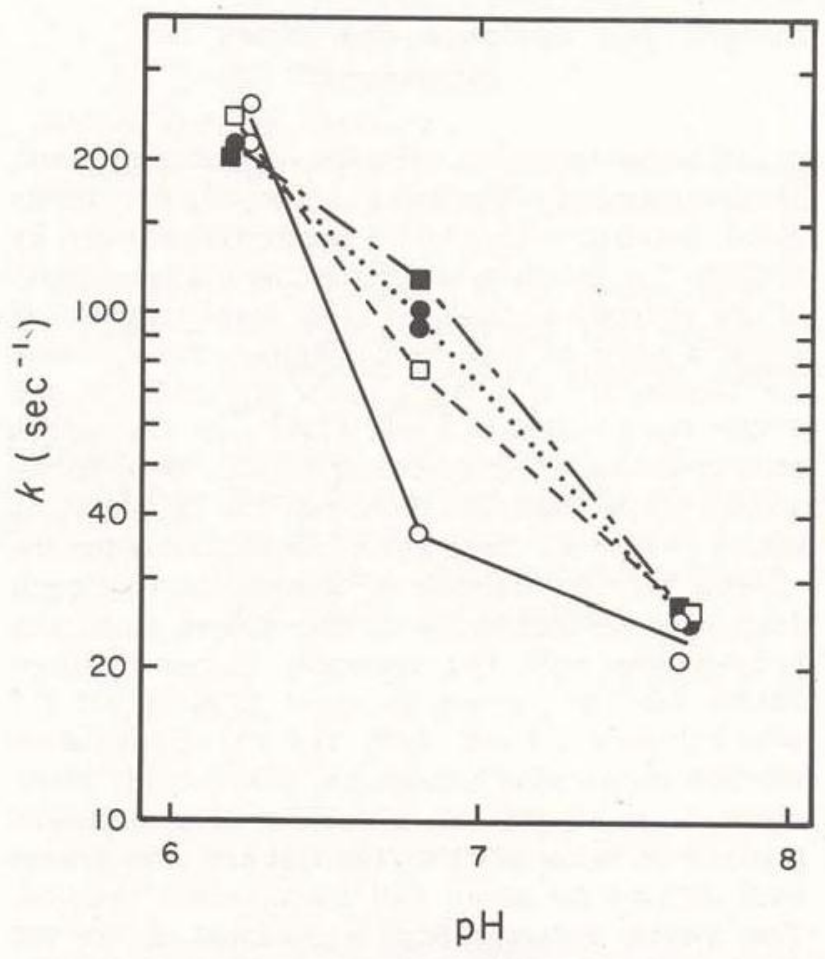

Fig. 2-Dependência do $\mathrm{pH}$ da velocidade de dissociação do oxigênio das hemoglobinas do Hoplias $\left(\mathrm{O}\right.$, ) e do Hoplerythrinus $(\square, \square)$ a $20^{\circ} \mathrm{C}$. Os símbolos abertos representam os dados obtidos em ausência de ATP e os fechados em presença de $1 \mathrm{mM}$ de ATP. A constante de velocidade de primeira ordem, K, é graficada em escala logarítmica em função do $\mathrm{pH}$.

A cinética da reação do monóxido de carbono com a hemoglobina do Hoplerythrinus foi medida sob as mesmas condições do Hoplias com resultados apresentando diferenças significativas. A heterogeneidade da cinética foi máxima ao $\mathrm{pH}$ baixo e cada fase respondendo por cerca da metade das reações observadas, porém a heterogeneidade decresce e desaparece se $\alpha \mathrm{pH}$ for aumentado. A constante de velocidade de segunda ordem foi esboçada na fig. 4. Ao pH 6,1, a fase rápida da reação tem uma velocidade constante, a qual é menor que metade da fase rápida da reação com a hemoglobina do Hoplias. A fase lenta tem uma velocidade semelhante à da hemoglobina do Hoplias, enquanto não é observado o efeito com ATP. Ao pH 8,2, a única constante de velocidade e próxima da mesma que foi obtida para a fase rápida ao $\mathrm{pH} 6,1$ e é apenas $30 \%$ mais rápida que a fase lenta observada com a hemcglobina do Hoplias neste $\mathrm{pH}$. Ao $\mathrm{pH}$ 7,1 , há um efeito definido ao ATP. Na ausência dele, a reação é cineticamente homogênea com uma velocidade igual à da fase lenta ao $\mathrm{pH}$ 6,1 . A adição de $1 \mathrm{mM}$ ATP baixa a velocidade da reação e induz heterogeneidade, embora a constante de reação para as duas fases difiram por um fator menor que 2. A origem da heterơgeneidade na reação destas hemoglobinas com o monóxido de carbono não está em nosso objetivo uma pcssível interpretação é que ela se reflete na heterogeneidade eletroforética da hemoglobina destes animais. Por outro lado, é estranho que cada uma das duas fases represente metade do total da reação. Isto é remanescente de alguns componentes simples das hemoglobinas do peixe, do qual tal heterogeneidade cinética provém a partir de uma heterogeneidade funcional das subunidades $\propto$ e $\beta$ (Bonaventura, et al. 1976; Pennelly, et al., 1978). Mais dados são necessários para uma escolha ser feita entre estas alternativas.

Em conclusão, este estudo tem demonstrado uma larga semelhança nas propriedades funcionais das hemoglobinas do Hoplias e Hoplerythrinus apesar das diferenças em seus componentes respiratórios sob condições hipóxica. Cineticamente, a maior diferença entre as duas hemoglobinas está nas velocidades da fase rápida de suas reações com monóxido de carbono. Fisiologicamente, a diferença na afinidade pelo oxigênio das duas hemoglobinas pode ser correlacionada com continuada respiração aquática pelos Hoplias e a mudança para respiração aérea pelos Hoplerythrinus em ambiente de baixo teor de oxigênio. 


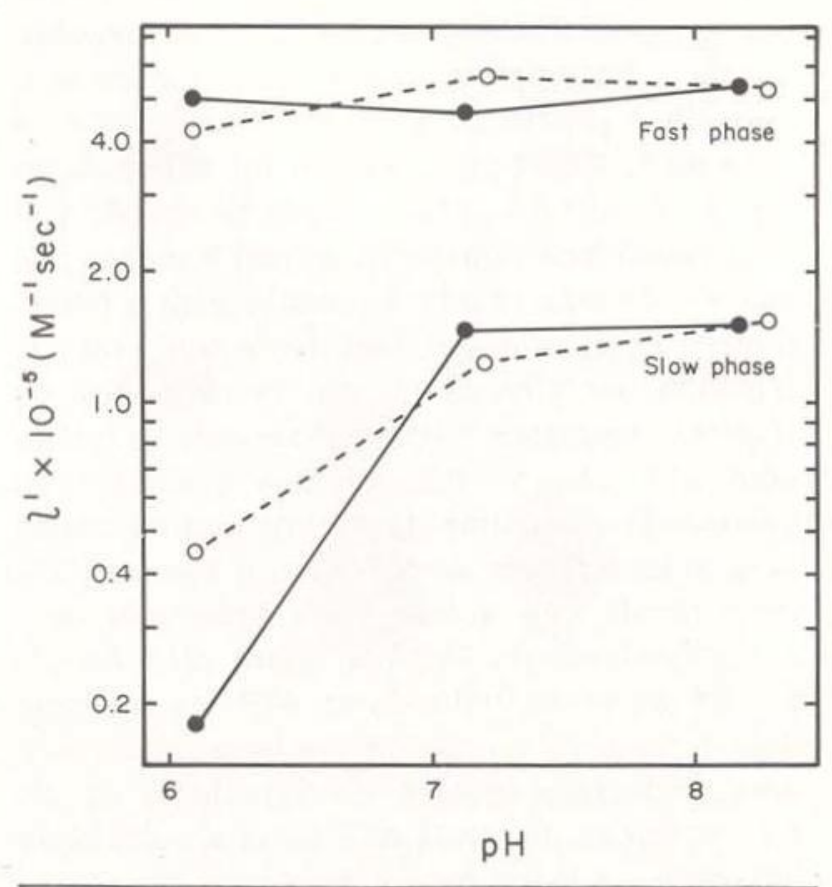

Fig. 3-Dependência do pH do constante de velocldade l' para as fases rápidas e lentas de combinação do monóxido de carbono com a hemoglobina do Hoplias em presença (•) ausência, O, de $1 \mathrm{mM}$ de ATP a $20^{\circ} \mathrm{C}$. As velocidades são desenhadas em escala logarítmica em função do $\mathrm{pH}$.

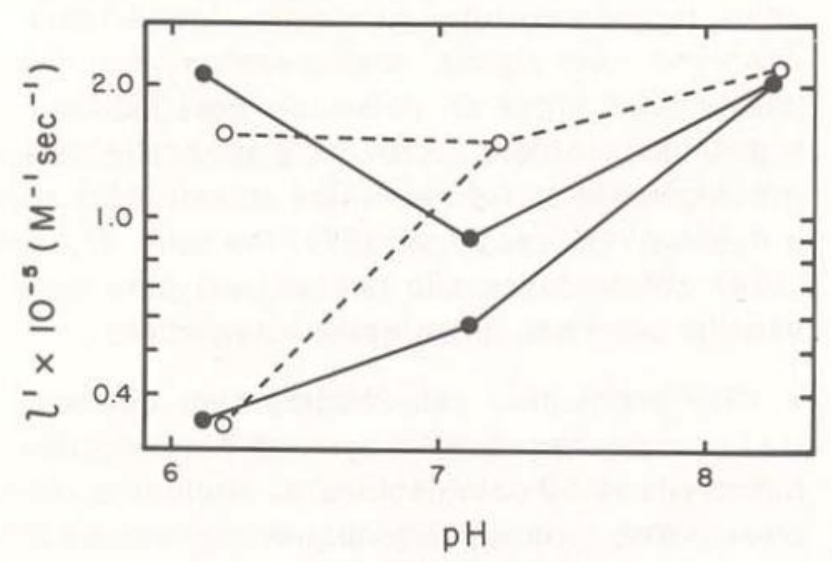

Fig. 4-Dependência do $\mathrm{pH}$ da constante de velocldade 1', para a combinação do monóxido de carbono com a hemoglobina do Hoplerythrinus a $20^{\circ} \mathrm{C}$, em presença ( $\bullet$ ), e ausência $(O)$ de $1 \mathrm{mM}$ de ATP. A constante de velocidade segunda ordem para está reação foi desenhada em escala logarítmica em função do pH \% Onde dois valores são dados para um único $\mathrm{pH}$, isto representa as constantes associadas com as fases separadas na reação bifásica.

\section{AgRADECIMENTOS}

Este trabalho foi patrocinado pela bolsa PCM75-06451 da NSF, e por outra da Norweigian Research Council for Science and the Humanities. Somos gratos aos Brasileiros por possibilitarem que R/V "Alpha Helix" entrasse no Amazonas e ao Capitão Clarck e sua tripulação por sua cooperação. Patrocínio adicional foi dado pelo NSF Frant PCM-76-06119 (A.R.) e pela University of Texas Research Institute. R. W. Noble is Established Investigator of the American Heart Association.

\section{SUMMARY}

Hemolysates from Hoplias malabaricus and Hoplerythrinus unitaeniatus show blurred hemoglobin patterns with 3 and 4 bands, respectively, by alkaline disc gel electrophoresis. The oxygen affinity of the stripped hemoglobin from Hoplerythrinus is about a third of that from Hoplias; the $P_{50}$ value for Hoplias $\mathrm{Hb}$ is about $1.3 \mathrm{~mm} \mathrm{Hg}(\mathrm{pH} 6.9$ and $20^{\circ} \mathrm{C}$.). The addition of $1 \mathrm{mM}$ ATP lowers the oxygen affinity of each hemoglobin 2.6 fold. Both hemoglobins show Root and Bohr effects; $\Delta \log \mathrm{P}_{50} / \Delta$ $\mathrm{pH}=-0.40$ for the stripped hemoglobins for the interval $\mathrm{pH} 7-8$. The rate of dissociation of oxygen from each hemoglobin is similar and is kinetically homogeneous with rate constants decreasing from 200-250 sec-1 at $\mathrm{pH} 6.2$ to about $25-26$ at $\mathrm{pH} 7.7$ with or without $1 \mathrm{mM}$ ATP. The CO combination reaction for Hoplias hemoglobin is kinetically heterogeneous at all $\mathrm{pH}$ values and for Hoplerythrinus hemoglobin below pH 7.5. The fast and slow phases each account for about half the observed reaction. The kinetic heterogeneity is maximal at low $\mathrm{pH}$ for both hemoglobins. The fast phase for Hoplias hemoglobin is more than twice that for Hoplerythrinus hemoglobins.

\section{BIBLIOGRAFIA}

Allen, D.W.; Guthe, K.F. \& Wyman, J.

1950 - Further studies on the oxygen equilibrium of hemoglobin. J. Biol. Chem., 187:393-410.

Bonaventura, C.; Sullivan, B.; Bonaventura, J. \& BRUNORI, M.

1976 - Spot hemoglobin. Studies on the Root Effect Hemoglobin of a Marine Teleost. J. Biol. Chem., 251:1871-1876. 
CARTer, G.S. \& BeAdle, L.C.

1931 - The Fauna of the Swamps of the Paraguayan Chaco in relation to its Environment. II. Respiratory adaptations in the Fishes. J. Linnean Society, $37: 327-368$.

DAvis, B.J.

1964 - Disc electrophoresis. II. Method and application to human serum proteins. Ann. N. Y. Acad. Sci., 121:404-427.

GARLICK, R.L.; DAVIS, B.J.; FARMER, M.; FYhn, H.J.; FyhN, U.E.H.; Noble, R.W.; POWERS, D.A.; RIGGS, A. \& WEBER, R.E.

1978 - Uma troca materno-fetal no equilíbrio de oxigênio das hemoglobinas dos caecilios viviparos Typholonectes compressicauda. Acta Amazonica 8(4) : Suplemento. (Este volume).

GIBSON, Q.H. \& MILNES, L.

1964 - Apparatus for Rapid and Sensitive Spectrophotometry. Biochem. J., 91 : 161-171.

NAGel, R.L.; WITtenberg, J.B. \& RANNEY, H.M.

1965 - Oxygen equilibria of the hemoglobinhaptoglobin complex. Biochim Biophys Acta, 100:286-289.

Noble, R.; PARkhurst, L.J. \& Gibson, Q.H.

1970 - The effect of $\mathrm{pH}$ on the reactions of oxygen and carbon monoxide the with hemoglobin of carp Cyprinus carpio. J. Biol. Chem., 245:6628-6633.
PENNelly, R.R.; RIGgs, A. \& Noble, R.W.

1978 - Kinetics and equilibria of squirrel fish hemoglobin; A Root effect hemoglobin complicated by Large subunit heterogeneity. Biochim. Biophys. Acta., (no prelo).

ORNSTEIN, L.

1964 - Disc electrophoresis. I. Background and theory. Ann. N. Y. Acad. Sci., 121: 321-349.

REISCHL, E.

1976 - The hemoglobins of the fresh-water teleost Hoplias malabarica (Bloch, 1794): Heterogeneity and Polymerization. Comp. Biochem. Physiol., 55B : 255-257.

RIGGS, A.F. \& WolbaCh, R.A.

1956 - Sulfhydryl groups and the structure of hemoglobin. J. Gen. Physiol., 39: 585:605.

Weber, K. \& OSBORN, M.

1969 - The reliability of molecular weight determinations by dodecyl sulfatepolyacrylamide electrophoresis. J. Biol. Chem., 244:4406-4412.

WILLMER, E.N.

1934 - Some observations on the respiration of Certain Tropical fresh-water fishes. J. Exp. Biol., II: 